CARDIOLOGY

\title{
Aortic Angulation and TAVR
}

\author{
Puneet Gandotra \\ Northwell Health, Bay Shore, NY, USA
}

Transcatheter valve technology has been a tremendous addition to the armamentarium of clinicians treating aortic stenosis. Treatment of inoperable, high-risk, intermediate-risk, and now low-risk patients with severe aortic stenosis using catheter-based valves is a significant shift in treatment paradigm.

As we perform these procedures in high volumes, we increasingly understand the variables that lead to success and failure. Multi detector CT (MDCT) has been an invaluable tool in annular sizing [1], measuring coronary heights and many other variables. The measurement of aortic angulation has been extremely useful in case planning. Measurement is performed in coronal projection and defined as an angle between the horizontal plane and the plane of the aortic annulus.

Traditionally, self-expanding valves have been limited to the aortic angulation of 70 degree or less and were exclusion criteria for clinical trials with SE trials [2]. Abramowitz et al. [3] found an inverse ratio between increased aortic root angulation and acute procedural success. Higher AA was associated with lower device success rates, higher postprocedural PVR rates, a higher need for post-dilation or a second valve, and a higher incidence of valve embolization following SE TAVR. In this study, AA of $<48$ and $>48$ degrees was used as a division of 2 groups.

\section{KARGER}

(C) 2018 S. Karger AG, Basel

E-Mail karger@karger.com

www.karger.com/crd
There was a rebuttal to this data by Popma et al. [4], which reviewed 3,578 patients from CoreValve clinical trials. With attention to best practices, AA was not shown to be independently associated with poor outcomes.

The study by Elmously et al. [5] builds on the available data for this topic. In a single center, 251 consecutive patients underwent TAVR with BE or SE valves. In previous studies, aortic angulation was treated as categories or groups, and in this retrospective study, AA was treated as a continuous variable. The paper emphasizes that there is loss of information with categorizing a continuous variable such as AA.

A higher rate of post dilation was noted with the SE valve, which was also seen in the study with Abramowitz et al. [3]. On univariate analysis, a trend toward more PVL with SE valve but not with multivariate analysis was observed. As seen in previous data sets [4], AA was not associated with increased procedure time.

This paper by Elmously et al. [5] adds to the growing consensus among the TAVR community that aortic angulation alone should not be contraindication to TAVR. Although there exist some trends toward increased procedural times and post dilation, using best practices, there appears to be no significant association with AA and procedural success.

Dr. Puneet Gandotra Northwell Health Bay Shore, NY 11803 (USA)

E-Mail puneetgandotra@gmail.com 


\section{References}

1 Jilaihawi H, Kashif M, Fontana G, et al: Crosssectional computed tomographic assessment improves accuracy of aortic annular sizing for transcatheter aortic valve replacement and reduces the incidence of paravalvular aortic regurgitation. J Am Coll Cardiol 2012;59:s1275s1286.

2 Adams DH, Popma JJ, Reardon MJ, et al: Transcatheter aortic-valve replacement with a self-expanding prosthesis. N Engl J Med 2014;370:1790-1798.

3 Abramowitz Y, Maeno Y, Chakravarty T, et al: Aortic angulation attenuates procedural success following self-expandable but not balloon-expandable TAVR. JACC Cardiovasc Imaging 2016;9:964-972.

4 Popma JJ, Reardon MJ, Yakubov SJ, et al: Safety and efficacy of self-expanding TAVR in patients with aortoventricular angulation. JACC Cardiovasc Imaging 2016;9:973981

5 Elmously A, Gray KD, Truong QA, Burshtein A, Wong SC, de Biasi AR, et al: Aortic angulation does not impact outcomes in self-expandable or balloon-expandable transcatheter aortic valve replacement. Cardiology 2018, DOI: $10.1159 / 000488933$. 